\title{
Delays in fault detection and isolation
}

\author{
Anton A. Stoorvogel*
}

\author{
Henrik Niemann ${ }^{\dagger}$
}

\author{
Ali Saberit
}

\section{Introduction}

Various types of faults arise in industrial processes owing to malfunction of internal components of a process as well as those in measurement sensors and control actuators attached to the process. Over the last three or four decades industrial automation has been increasingly fueled by various technological developments including the availability of highly complex electronic equipment and the overwhelming progress in computer technology. This has led not only to the development of complex control systems but also to higher demand of reliable and secure control systems. Thus it has become imperative that any fault that occurs be detected and identified automatically without severely disturbing the yield the process generates. This has stimulated over the last two decades an extensive study of fault detection and isolation methods.

As discussed in a survey paper by Willsky, [5], one faces three different types of tasks or layers in the area of fault detection and isolation, (1) fault detection, (2) fault isolation, and (3) fault estimation. Fault detection consists of designing a residual generator that produces a residual signal enabling one to make a binary decision as to whether a fault occurred or not. Fault isolation imposes a stronger requirement. When one or more faults occur, the residual signal must enable us not only to detect that there are faults occurring in the system, but it must also enable us to identify (isolate) which faults have occurred. Finally, fault estimation is the determination of the extent of failure. The latter is done by trying to reconstruct the fault signals.

A number of fundamental problems that arise in fault estimation, i.e. in estimating the fault signals have been studied recently by us [ 1]. We also studied fault detection and fault isolation in the paper [2].

However, an issue which, as far as we know, has not been studied in this context is to estimate and minimize the time it takes after a fault occurs to actually detect the fault on the basis of the residual signal.

For discrete-time systems, the notion of using a fixed delay in estimating a fault signal has been introduced in [1]. That is, at time step $k$, one obtains the estimate of the fault signal at $k-\ell$ where $\ell$ is a fixed nonnegative integer. This clearly weakens the solvability conditions. In [2] we looked into using this delay for fault detection and isolation and we noted this does not weaken the solvability conditions. However, this is due to a fundamental aspect of our problem formulation. In fault detection we required the residual signal to be nonzero if a fault occured and otherwise to be zero. However, we did not impose any constraint on the time between the occurence of a fault and the moment that the residual signal becomes nonzero indicating that a fault has occured. Obviously in many applications this delay in detecting a fault can be quite dangerous and therefore this delay should be as small as possible.

In this paper we show that if fault detection and isolation is possible for continuous time systems, then we can make the delay arbitrary small. However, in discrete time this delay will in the worst case be $n$ timesteps for a system of McMillan degree $n$. We formulate in this paper only the problems of exact fault detection and isolation. In [2] we extended these results to generic and almost fault detection and isolation. The results of this paper also cover these cases but the presentation of these results in this conference paper are not possible due to space limitations. The following definitions will be needed later on:

Definition 1.1 Consider a linear system $\Sigma$ characterized by a quadruple $(A, B, C, D)$. The strongly controllable subspace $\&(A, B, C, D)$ is defined as the smallest subspace of $\mathbb{R}^{n}$ which is $(A+K C)$-invariant and containing $\operatorname{im}(B+K D)$.

Definition 1.2 Consider a linear system $\Sigma$ characterized by a quadruple $(A, B, C, D)$. The system is said to be left invertible if the transfer matrix of the system has a left inverse (not necessarily proper or stable).

Definition 1.3 Consider a linear system $\Sigma$ characterized by a quadruple $(A, B, C, D)$. The infinite zeros of this system are equal to the infinite zeros of the corresponding transfer matrix.

Note that the order of the infinite zeros are determined through the Smith-McMillan form at infinity of the transfer matrix.

\footnotetext{
* Department of Mathematics and Computing Science, Eindhoven Univ. of Technology, P.O. Box 513, 5600 MB Eindhoven and Department of Information Technology and Systems, Delft Univ. of Technology, P.O. Box 5031,2600 GA Delft, The Netherlands, E-mail: a.a.stoorvogel@tue.nl

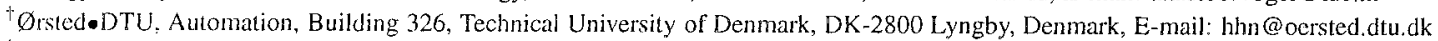

*School of Electrical Engineering and Computer Science, Washington State University, Pullman, WA 99164-2752, U.S.A., E-mail: saberi@eecs.wsu.edu
} 


\section{Problem Formulations and Main Results}

Consider the following state space description for a plant or a system given by

$$
\Sigma:\left\{\begin{aligned}
\sigma x & =A x+\sum_{j=1}^{n} E_{j} d_{j}+\sum_{i=1}^{k} L_{i} f_{i} \\
& =A x+E d+L_{f} f \\
y & =C x+\sum_{j=1}^{m} D_{d, j} d_{j}+\sum_{i=1}^{k} D_{f, i} f_{i} \\
& =C x+D_{d} d+D_{f} f
\end{aligned}\right.
$$

where $\sigma$ is an operator indicating the time derivation $\frac{d}{d t}$ for continuous-time systems and a forward unit time shift for discrete-time systems. Also, $x \in \mathbb{R}^{n}$ is the state vector, $d \in \mathbb{R}^{m}$ is a disturbance signal vector, and $y \in \mathbb{R}^{p}$ is the measurement vector. Furthermore, $f_{i}$ signifies the $i$-th fault for each $i=1,2, \ldots, k$. The coefficient matrices $L_{i}$ and $D_{f, i}$ are referred to in the literature as failure signatures associated with the $i$-th fault, while $f_{i}$ itself is called the $i$-th fault signal. Obviously, the failure signatures $L_{i}$ and $D_{f, i}$ depend on the physics of the given system. The fault signal vector $f \in \mathbb{R}^{k}$ is a collection of fault signals $f_{i}, i=1,2, \ldots, k$, into a vector. We will sometimes need to refer to the fault signal $\tilde{f}_{i} \in \mathbb{R}^{k}$ which is a vector with all elements equal to zero except for the $i$ 'th position where it is equal to $f_{i}$. Because there is no possibility for confusion and to simplify notation we will denote both $\tilde{f}_{i}$ and $f_{i}$ by $f_{i}$. It is always clear from the context which interpretation we are using.

In modeling a given plant by the system (2.1), we assume that all the fault signals $f_{i}, i=1,2, \ldots, k$, are quite arbitrary and that no information is known regarding their characteristics. That is, none of the signals $i=1,2, \ldots, k$, are constrained to belong to any special class of functions.

We now proceed to formulate certain fault detection and isolation problems. The fault detection setup we follow here is shown in the following figure:

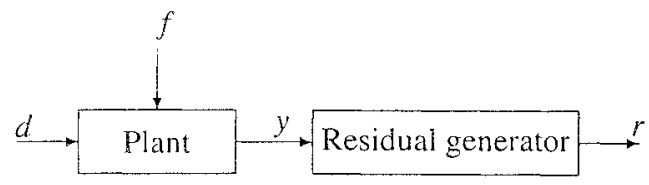

Let the residual signal $r$ be given by

$$
r=H y=\Psi(d, f)
$$

where $r$ is a time function that takes values in $\mathbb{R}^{q}$. In general, we might have to take $H$ to be a nonlinear boundedinput, bounded-output stable operator which makes $\Psi$ also a nonlinear operator mapping disturbances and faults to a residual signal $r$. Of course, if $H$ is linear, then there exist transfer matrices $G_{r f}$ and $G_{r d}$ such that

$$
r=G_{r f} f+G_{r d} d \text {. }
$$

One of the basic issues that concerns fault detection and isolation is whether one can achieve such a detection and isolation when the disturbance $d$ affects the system. This points out a need to have a residual generator which is insensitive to the external disturbance $d$. That is, we need that

$$
\Psi(d, f)=\Psi(0, f)
$$

for all disturbances $d$ and all fault signals $f$ or at least that the dependence of $r$ on $d$ is arbitrarily small with respect to some specified norm. If $H$ is linear then this implies that we impose that the transfer matrix $G_{r d}$ is zero or arbitrarily small in some specific norm.

Before we proceed, we need to consider certain modeling aspects. In a given situation, there exists always a number of possible faults. Some of these individual faults might occur simultaneously at any given time and others cannot. The tasks of fault detection and isolation depend on which faults can occur simultaneously and which cannot. In this paper we consider the two extreme cases: either faults can occur simultaneously (without any restrictions) or faults cannot occur simultaneously, i.e. two faults never occur simultaneously. Moreover, in the latter case, faults occur sufficiently apart in time so that at any given time at most one fault affects the measurement signal.

The subsection 2.1 defines the basic problems that correspond to fault detection, the subsection 2.2 defines the basic problems that correspond to fault isolation.

\subsection{Fault detection}

As mentioned in the introduction, the task of fault detection consists of designing a residual generator that produces a residual signal enabling one to make a binary decision as to whether a fault or faults occurred or not.

Problem 2.1 Consider the system given in (2.1). The problem of (exact) fault detection of a set of multiple faults $f$ with signature matrices $L_{f}$ and $D_{f}$ is defined as the problem of finding, if existent, a bounded-input boundedoutput stable residual generator $H$ whose output is a scalar residual signal $r=\Psi(d, f)$ such that

(i) $\Psi(d, 0)=0$ for all disturbances $d$.

(ii) $\Psi(d, f) \neq 0$ for all faults $f \neq 0$ and all disturbances $d$.

We say that the set of multiple faults with signature matrices $L_{f}$ and $D_{f}$ is exactly detectable if the above problem is solvable for it. 


\subsection{Fault Isolation}

The task of fault detection exists only when there is a possibility of multiple faults occurring. In that case, in addition to detecting that a fault or faults occurred, one has to identify as to what individual fault or faults have occurred. Let us also emphasize that fault isolation can be sought at different levels. The most demanding level is the one that seeks to identify each and every individual fault that occurred. In certain aspects of engineering, one may not need to identify each and every individual fault that occurred. Perhaps, one can classify all possible faults into certain classes. Then, one needs simply to ascertain that a fault or faults belonging to a particular class or classes have occurred.

In connection with fault isolation, it is important to recognize that the detectability of a set of faults must be ascertained before one faces the task of fault isolation.

We first consider the case when we need to identify each and every individual fault that occurred. It is easy now to recognize that the task of isolating or identifying every individual fault requires that we generate for each individual fault signal $f_{i}$ a dedicated residual signal $r_{i}$ such that $r_{i}$ would be insensitive to all disturbances and all vector faults for which $f_{i}$ is identical to zero while it is sensitive to all vector faults for which $f_{i}$ is not identical to zero, It is clear from this discussion that, for the task of individual fault isolation, the dimension of residual vector can always be taken the same as the dimension of fault vector $f$ itself.

We can now have the following precise formulation of fault isolation problem that seeks to identify each individual fault that occurred.

Problem 2.2 Consider the system given in (2.1) under the simultaneous occurrence property. Then, the problem of (exact) individual fault isolation for a set of faults $f$ with signature matrices $L_{f}$ and $D_{f}$ is defined as a problem of finding, if existent, a bounded-input bounded-output stable residual generator $H$ which generates a residual vector $r=\Psi(d, f)$ such that for any fault $f_{i}, i=1,2, \ldots, k$, there exists a dedicated component $r_{i}$ of $r$ and the operator $\Psi_{i}$ from $d$ and $f$ to $r_{i}$ has the following properties:

- $\Psi_{i}(d, f)=0$ for any disturbance $d$ and any fault $f$ such that $f_{i}$ is identical to zero.

- $\Psi_{i}(d, f) \neq 0$ for any disturbance $d$ and any fault $f$ such that $f_{i}$ is not identical to zero.

The set of faults $f$ with signature matrices $L_{f}$ and $D_{f}$ is said to be individually identifiable if the problem of individual fault isolation is solvable.

\subsection{Delays in fault detection and isolation}

For ease of presentation we define the class of all causal signals as $\wp_{c}$, i.e. $f \in \diamond_{c}$ if and only if $f(t)=0$ for all $i<0$.
Assume we have a residual generator $H$ yielding a residual signal $r$. We have:

$$
r=H y=\Psi(d, f)
$$

Assume that this residual generator achieves exact fault detection. That is,

- $\Psi(d, 0)=0$ for all $d$

- $\Psi(d, f)$ is nonzero for all $d$ and for all nonzero $f$.

Then we can define the detection delay $d_{H}$ :

$$
\left.d_{H}:=\sup _{\substack{f \in s_{c} \\ f \neq 0}} \sup _{\substack{0 \\ f \neq 0}} \operatorname{in} \mid r(t) \neq 0\right\}
$$

Note that $d_{H} \geqslant 0$ by causality of the system. Theoretically $d_{H}$ could be equal to $+\infty$ but in any normal design this detection delay will be finite. $d_{H} H$ gives the worst case delay in detecting a fault and obviously we would like to minimize this delay.

For fault isolation we again consider a residual generator $H$ and define $\Psi$ according to (2.3). Assume that this residual generator achicves exact fault isolation. Then we can again define the isolation delay

$$
i_{H}:=\sup _{i=1, \ldots, m} \sup _{\substack{f \in S_{c} \\ f_{i} \neq 0}} \sup \inf \left\{t \mid r_{i}(t) \neq 0\right\}
$$

Note that again $i_{H} \geqslant 0$ by causality of the system and theoretically could be equal to $+\infty$. $i_{H}$ gives the worst case delay in detecting and isolating a fault and obviously we would like to minimize this delay.

The following theorems gives a positive result for continuous time systems in the sense that we can always guarantee $i_{H}=0$ and $d_{H}=0$. These results are independent of the fact whether or not fault can occur simultaneously or not.

Theorem 2.3 Assume that fault detection is possible for a continuous-time system of the form (2.1). Then there also exists a residual generator which achieves fault detection and is such that $d_{H}=0$.

Theorem 2.4 Assume that fault isolation is possible for a continuous-time system of the form (2.1). Then there also exists a residual generator which achieves fault isolation and is such that $i_{H}=0$.

However, for discrete-time systems the situation is quite different. In order to present these results we make a decomposition of the state space $x=x_{1} \oplus x_{2}$ with $x_{1}=$ $s^{*}\left(A, E, C, D_{d}\right)$ and a decomposition of the output space $y=y_{1} \oplus y_{2}$ with $y_{1}=C \xi^{*}\left(A, E, C, D_{d}\right)+\operatorname{im} D_{d}$. 
For details we refer to $[3,4]$. With respect to this decomposition the system takes the following form for suitably chosen $K_{1}$ and $K_{2}$ :

$$
\begin{aligned}
\dot{x}= & \left(\begin{array}{cc}
A_{11} & A_{12} \\
0 & A_{22}
\end{array}\right) x+\left(\begin{array}{c}
E_{1} \\
0
\end{array}\right) d+\left(\begin{array}{l}
L_{1} \\
L_{2}
\end{array}\right) f \\
& +\left(\begin{array}{c}
K_{1} \\
K_{2}
\end{array}\right) y \\
y & =\left(\begin{array}{cc}
C_{11} & C_{12} \\
0 & C_{22}
\end{array}\right) x+\left(\begin{array}{c}
D_{d, 1} \\
0
\end{array}\right) d+\left(\begin{array}{l}
D_{f, 1} \\
D_{f, 2}
\end{array}\right) f
\end{aligned}
$$

We denote the columns of $L_{2}$ and $D_{f, 2}$ by $L_{2, i}$ and $D_{f, 2, i}$ respectively.

Theorem 2.5 Consider the system (2.4). We have the following results:

- Assune faults can occur simultaneously. Fault derection is possible if and only if the system $\left(A_{22}, L_{2}, C_{22}, D_{/ 2}\right)$ is left invertible. Moreover $d_{H}$ is equal to the maximal order of the infinite zeros of the system $\left(A_{22}, L_{2}, C_{22}, D_{f, 2}\right)$.

- Assume faults cannot occur simultaneously. Fault detection is possible if and only if the transfer matrix of the system $\left(A_{22}, L_{2}, C_{22}, D_{f, 2}\right)$ has no columns identical to zero. Moreover $d_{H}$ is equal to the maximal order of the infinite zeros of the systems $\left(A_{22}, L_{2, i}, C_{22}, D_{f, 2, i}\right)$ for $i=1, \ldots k$.

Theorem 2.6 Consider the system (2.4). We have the following results:

- Assume faults can occur simultaneously. Fault isolation is possible if and only if the system $\left(A_{22}, L_{2}, C_{22}, D_{f, 2}\right)$ is left invertible. Moreover $i_{H}$ is equal to the maximal order of the infinite zeros of the system $\left(A_{22}, L_{2}, C_{22}, D_{f, 2}\right)$.

- Assume faults cannot occur simultaneously. Fault isolation is possible if and only if the system

$$
\left(A_{22},\left(\begin{array}{ll}
L_{2, i} & L_{2, j}
\end{array}\right), C_{22},\left(\begin{array}{ll}
D_{f, 2, i} & D_{f, 2, j}
\end{array}\right)\right)
$$

is left invertible for all $i, j=1, \ldots, k$ with $i \neq j$. Moreover $d_{H}$ is equal to the maximal order of the infinite zeros of the systems (2.5) for $i, j=1, \ldots, k$ with $i \neq j$.

Note that from the above two theorems it is clear that if faults can occur simultaneously then fault detection is possible if and only if fault isolation is possible and $d_{H}=i_{H}$. On the other hand, if faults cannot occur simultaneously then the solvability conditions for fault detection and isolation are different and in general $d_{H} \neq i_{H}$.

The following example illustrates that $i_{H}$ and $d_{H}$ can be different and also that the delay can be influenced by whether or not faults can occur simultaneously.
Example 2.7 Consider the following system:

$$
\begin{aligned}
\dot{x}= & \left(\begin{array}{cccc}
-2 & 0 & 0 & 0 \\
0 & -1 & 0 & 0 \\
0 & 0 & -3 & 0 \\
0 & 0 & 0 & -4
\end{array}\right) x+\left(\begin{array}{l}
1 \\
0 \\
0 \\
0
\end{array}\right) d \\
& +\left(\begin{array}{ccc}
1 & -2 & 3 \\
1 & 0 & 0 \\
0 & 1 & 0 \\
0 & 0 & 1
\end{array}\right) f \\
y= & \left(\begin{array}{cccc}
1 & 2 & 1 & 3 \\
0 & 1 & 0 & 0 \\
0 & 0 & 1 & 0 \\
0 & 0 & 0 & 1
\end{array}\right) x+\left(\begin{array}{l}
1 \\
0 \\
0 \\
0
\end{array}\right) d \\
& +\left(\begin{array}{lll}
2 & 3 & 8 \\
0 & 1 & 0 \\
0 & 0 & 1 \\
0 & 0 & 0
\end{array}\right) f
\end{aligned}
$$

The decomposition as presented in (2.5) is quite trivial in this case with $K_{1}$ and $K_{2}$ equal to zero and $\chi_{1}$ and $y_{1}$ equal to the one dimensional subspace of the state and output space respectively which is spanned by the first unit vector. We obtain:

- If faults can occur simultaneously then fault detection and isolation is possible and $d_{H}=i_{H}=3$. We can see that a delay $\dot{i}_{H}$ is inevitable by condidering the case:

$$
\begin{aligned}
& f_{1}(k)=\left\{\begin{array}{ll}
1 & k=0 \\
0 & k \neq 0
\end{array} \quad f_{2}(k)= \begin{cases}-1 & k=1 \\
0 & k \neq 0\end{cases} \right. \\
& f_{3}(k)= \begin{cases}1 & k=2 \\
0 & k \neq 0\end{cases}
\end{aligned}
$$

while

$$
d(k)=-\left(\begin{array}{lll}
2 & 3 & 8
\end{array}\right) f-\left(\begin{array}{llll}
1 & 2 & 1 & 3
\end{array}\right) x
$$

which yields $y(0)=y(1)=y(2)=0$ and only $y(3)=1$ gives the first indication that a fault has occured.

- If faults cannot occur simultaneously then fault detection and isolation is possible and $d_{H}=1$ while $i_{H}=2$. 


\section{Conclusion}

This paper studies an aspect of detection and isolation delays which has been ignored in many papers in this area. Surprising since it is highly relevant from a practical point of view. In continuous-time systems this is not a serious matter since we can always guarantee an arbitrary small delay and nearly all designs in the literature yield this arbitrary small delay. However, for discrete-time systems the situation is different and by designing specifically to reduce the detection or isolation delay we can get better results compared to most designs as available in the literature.

\section{References}

[1] H. Niemann, A. Saberi, A. Stoorvogel, And P. SANNUTi, "Exact, almost and delayed fault detec- tion - an observer based approach", Int. J. Robust \& Nonlinear Control, 9(4), 1999, pp. 215-238.

[2] A. Saberi, A.A. Stoorvogel, P. Sannuti, And H.H. NIEMANN, "Fundamental problems in fault detection and identification", Int. J. Robust \& Nonlinear Control, 10(14), 2000, pp. 1209-1236.

[3] P. Sannuti And A. SAberi, "Special coordinate basis for multivariable linear systems - finite and infinite zero structure, squaring down and decoupling", Int. J. Contr., 45(5), 1987, pp. 1655-1704.

[4] H.L. Trentelman, A.A. Stoorvogel, And M.L.J. HAUTUS, Linear multivariable systems, Communication and Control Engineering Series, Springer Verlag, 2001.

[5] A.S. WILLSKY, "A survey of design methods for failure detection in dynamic systems", Automatica, 12, 1976, pp. 601-611. 